\title{
Unvermeidbare Ungleichheiten? Altagsweltliche Ungleichheitsdeutungen zwischen sozialer Konstruktion und gesellschaftlicher Notwendigkeit
}

\author{
\begin{tabular}{ll}
\cline { 2 - 2 } Matrick Sournal Article \\
Pachweh
\end{tabular} $\begin{aligned} & \text { Patrick Sachweh: Unvermeidbare Ungleichheiten? Alltagsweltliche Ungleichheitsdeutungen zwischen sozialer Konstruktion und } \\
& \text { gesellschaftlicher Notwendigkeit. In: Berliner Journal für Soziologie 21(4), 561-586 (2011). Springer VS }\end{aligned}$ \\ The original publication is available at the publisher's web site: http://dx.doi.org/10.1007/s11609-011-0168-6. \\ The MPIfG Journal Articles series features articles by MPIfG researchers and visiting scholars published in peer-reviewed journals. \\ Max Planck Institute for the Study of Societies (MPIfG) Cologne | www.mpifg.de
}

\begin{abstract}
Zusammenfassung: Der Artikel rekonstruiert einen unausgesprochenen „aufklärerischen Common Sense" der Ungleichheitssoziologie, demzufolge sich mit dem Übergang zur Moderne bei den Menschen ein Bewusstsein der gesellschaftlichen Ursachen sozialer Ungleichheitsverhältnisse herausgebildet habe und diese daher legitimationsbedürftig seien. Es wird gefragt, inwiefern dies eine realistische Annahme über die alläglichen Ungleichheitsdeutungen der Gesellschaftsmitglieder ist. Auf der Basis qualitativer Interviews mit Menschen in privilegierten und benachteiligten Lagen wird ein Deutungsmuster der „Unvermeidbarkeit sozialer Ungleichheit“ rekonstruiert, das Ungleichheit nicht als von Menschen gemachtes soziales Konstrukt, sondern als eine unausweichliche Notwendigkeit gesellschaftlicher Ordnung ansieht. Zwar existiert parallel ein Deutungsmuster der „Herkunftsbedingtheit sozialer Ungleichheit“, das die sozialen Konstruktionsprozesse von Ungleichheit in den Vordergrund rückt. Durch die Koexistenz beider Deutungsmuster im Alltagsbewusstsein der Befragten wird jedoch das ungleichheitskritische Potenzial der Herkunftsdeutung unterlaufen.
\end{abstract}

Schlüsselwörter: Soziale Ungleichheit · Sozialstruktur · Wahrnehmung · Deutungsmuster · Theorie

\section{Inevitable inequalities? Contingency and necessity in lay explanations of stratification}

\begin{abstract}
The article sketches a tacit "enlightened common sense" within stratification research according to which citizens in modern societies regard inequality as being caused by social factors and therefore in need of legitimation. The paper asks whether this is a plausible assumption about lay interpretations of stratification. Based on qualitative interviews with interviewees in privileged and disadvantaged social positions, the article reconstructs an interpretive scheme of the "inevitability of inequality" which regards inequality as an inescapable necessity of every social order and not as a contingent societal construct. Yet, another interpretive scheme exists that emphasizes the influence of social origin and thereby points to the social roots of stratifica-
\end{abstract}

(C) VS Verlag für Sozialwissenschaften 2011

P. Sachweh $(\bowtie)$

Institut für Gesellschafts- und Politikanalyse, Goethe-Universität Frankfurt a. M.

Robert-Mayer-Straße 5, 60054 Frankfurt a. M., Deutschland

E-Mail: sachweh@soz.uni-frankfurt.de 
tion. Empirically, however, both interpretive schemes coexist within the consciousness of the interviewees. The potential for the criticism of inequality is thus circumscribed.

Keywords: Social inequality $\cdot$ Social structure $\cdot$ Perception $\cdot$ Interpretive frame $\cdot$ Theory

\section{Inégalités inévitables? Les interprétations quotidiennes des inégalités entre construction sociale et nécessité sociale}

Résumé: Cet article reconstruit le «sens commun éclairé» tacite de la sociologie des inégalités selon lequel avec l'avènement de l'époque moderne les individus auraient pris conscience des causes sociales des inégalités sociales, créant ainsi un besoin de légitimation de ces inégalités. La question posée est de savoir dans quelle mesure cette hypothèse concernant les interprétations quotidiennes des inégalités par les membres de la société est réaliste. À partir d'entretiens qualitatifs avec des personnes en situation privilégiée et défavorisée, nous reconstruisons un schéma interprétatif de «l'inévitabilité de l'inégalité sociale» selon lequel les inégalités ne sont pas le produit de l'action humaine mais une nécessité inhérente à l'ordre social. Il existe certes un schéma interprétatif concurrent de «l'inégalité sociale liée à l'origine» qui met en avant les processus de construction sociale des inégalités. La coexistence de ces deux schémas interprétatifs dans la conscience quotidienne des personnes interrogées neutralise cependant le potentiel critique de l'interprétation basée sur les origines.

Mots-clés: Inégalité sociale · Structure sociale · Perception · Schéma interprétatif - Théorie

\section{Einleitung ${ }^{1}$}

Zu den Grundannahmen der Ungleichheitssoziologie zählt die Auffassung, dass in modernen Gesellschaften soziale Ungleichheit nicht mehr durch den Verweis auf eine natürliche Ordnung gerechtfertigt werden könne. Mit dem Übergang zur Moderne, so die Annahme, sei die gesellschaftliche Bedingtheit sozialer Ungleichheitsverhältnisse ins Bewusstsein der Menschen gelangt (Koller 1987). Insbesondere der Aufstieg des Gleichheitsideals im Zuge der Aufklärung sowie die Französische Revolution hätten zu weitreichenden Veränderungen der Wahrnehmung sozialer Ungleichheit seitens der Gesellschaftsmitglieder geführt (Parsons 1970). Ungleichheit gelte den Menschen in der Moderne nicht länger als gottgegebene oder natürliche Tatsache, sondern als Produkt menschlichen Handelns - und somit als grundsätzlich veränderbar und legitimationsbedürftig (Dahrendorf 1974; Eder 1990). Diese Annahme bildet den Kern eines Gedankengangs, den ich als den aufklärerischen Common Sense der Ungleichheitsforschung bezeichne. Dieser bezieht sich keineswegs nur auf die geistesgeschichtliche Thematisierung von Ungleichheit durch Philosophen oder Intellektuelle, sondern stellt zugleich eine implizite soziologische Annahme über das Alltagswissen breiter Bevölkerungskreise dar.

1 Eine erste Fassung dieses Artikels entstand während eines Postdoc-Aufenthaltes am MaxPlanck-Institut für Gesellschaftsforschung in Köln im akademischen Jahr 2009/2010. Ich danke den Mitgliedern der Forschergruppe Soziologie des Marktes am MPIfG sowie den Herausgebern und Gutachtern dieser Zeitschrift für hilfreiche Kommentare und Anmerkungen zu früheren Versionen dieses Aufsatzes. 
Der vorliegende Artikel fragt, ob diese stillschweigende Annahme über die alltäglichen Ungleichheitsdeutungen der Menschen realistisch ist. Im Einzelnen werden zwei Fragen gestellt: 1) Wird Ungleichheit in modernen Gesellschaften - hier: der zeitgenössischen deutschen Gesellschaft - von den Menschen tatsächlich vorrangig als sozial bedingt wahrgenommen, oder existieren noch andere Erklärungsmuster für Ungleichheit? 2) Welche Implikationen ergeben sich aus diesen Wahrnehmungen für die normativen Orientierungen der Menschen - und damit die Legitimation sozialer Ungleichheit? Führen Deutungsmuster, die soziale Ungleichheitsursachen betonen, auch zu ungleichheitskritischen normativen Orientierungen, wie vom aufklärerischen Hintergrundkonsens angenommen?

Anhand leitfadengestützter qualitativer Interviews mit Angehörigen unterschiedlicher sozialer Klassen in Deutschland wird gezeigt, dass zwischen den theoretischen Erwartungen des aufklärerischen Common Sense bezüglich der Ungleichheitsdeutungen der Menschen und ihrem tatsächlichen Alltagswissen eine Diskrepanz besteht. Empirisch lassen sich auf Basis der qualitativen Interviewdaten unterschiedliche Deutungsmuster sozialer Ungleichheit finden. Zwar existiert ein Deutungsmuster der Herkunftsbedingtheit sozialer Ungleichheit, das - wie vom aufklärerischen Common Sense erwartet - soziale Umstände zur Erklärung gesellschaftlicher Ungleichheitsverhältnisse in den Vordergrund rückt. Parallel dazu ist jedoch auch eine Persistenz „,voraufklärerischer“ Erklärungsmomente sozialer Ungleichheit zu konstatieren, die sich zu einem Deutungsmuster der Unvermeidbarkeit sozialer Ungleichheit verdichten lassen. In dessen Rahmen wird Ungleichheit nicht als Produkt menschlichen Handelns angesehen, sondern als eine nahezu unausweichliche Notwendigkeit gesellschaftlicher Ordnung. Die Interviews zeigen, dass beide Deutungsmuster im Alltagsbewusstsein der Befragten koexistieren, von ihnen kombiniert und - trotz ihrer latenten Widersprüchlichkeit - miteinander in Einklang gebracht werden. Darüber hinaus zeigt sich, dass mit der Herkunftsdeutung tatsächlich ungleichheitskritischere Deutungen einhergehen, die jedoch durch ihre Koexistenz mit der Unvermeidbarkeitsdeutung unterlaufen werden.

Vor der Darstellung dieser empirischen Befunde werden die theoretischen Hintergrundannahmen der Untersuchung expliziert. Zunächst wird der aufklärerische Common Sense der Ungleichheitsforschung rekonstruiert (2) und auf der Basis des Konzepts des sozialen Deutungsmusters ein theoretischer Rahmen für die empirische Untersuchung alltagsweltlicher Ungleichheitsdeutungen entwickelt (3). Nach einer Beschreibung der methodischen Vorgehensweise (4) werden im empirischen Teil (5) dann die beiden Deutungsmuster sozialer Ungleichheit herausgearbeitet, ihre Koexistenz in den Alltagsdeutungen der Befragten skizziert und nach ihren Implikationen für die normativen Orientierungen der Interviewpartner gefragt. Abschließend werden die Implikationen der Befunde sowohl für die ungleichheitssoziologische Theorie wie auch die empirische Forschung diskutiert (6).

\section{Der aufklärerische Common Sense der Ungleichheitsforschung}

Den Kern des eingangs skizzierten aufklärerischen Common Sense bildet die Annahme, dass mit dem Übergang zur Moderne zunehmend die gesellschaftlichen Ursachen sozia- 
ler Ungleichheitsverhältnisse ins Bewusstsein der Gesellschaftsmitglieder treten. Die vor dem Beginn der Aufklärung dominanten Sichtweisen erklärten soziale Ungleichheit meist mit den natürlichen (d. h. angeborenen) Unterschieden der Menschen oder der göttlichen Vorsehung. So behauptete etwa Aristoteles, es gebe eine Kongruenz von natürlichen und sozialen Unterschieden: „Es ist also klar, daß es von Natur Freie und Sklaven gibt und daß das Dienen für diese zuträglich und gerecht ist... Desgleichen ist das Verhältnis des Männlichen zum Weiblichen von Natur so, daß das eine besser, das andere geringer ist, und das eine regiert und das andere regiert wird..." (zitiert nach Dahrendorf 1974, S. 355, Hervorhebungen von Dahrendorf). Während des Mittelalters wurde Ungleichheit zudem auch als Ausdruck einer göttlichen Ordnung angesehen, etwa in der christlichen Konzeption einer ,great chain of being“. Die gesellschaftliche Ordnung wurde als eine Hierarchie abgestufter Positionen begriffen, in der jedem Wesen ein Platz gemäß seiner göttlichen Bestimmung zukam und in der alle Elemente zur Erfüllung des göttlichen Willens beitrugen (Bottero 2005, S. 15 ff.). ${ }^{2}$

Die Dominanz derartiger Vorstellungen wurde während des 18. Jahrhunderts aufgebrochen, als sich im Zuge der Aufklärung die Idee der naturrechtlichen Gleichheit aller Menschen verbreitete (Eder 1990; Parsons 1970) und - nicht zuletzt als Folge der Französischen Revolution - die gesellschaftlichen Verhältnisse zunehmend als gestaltbar erschienen (Dahrendorf 1974; Dux 2008, S. 64 ff.; Kreckel 2004, S. 27 f.). Damit stellte sich auch die Frage nach den Ursachen der Ungleichheit neu. „Wenn die Menschen von Natur gleich sind“, so Dahrendorf (1974, S. 357 f.), ,dann können die sozialen Ungleichheiten nicht natur- oder gottgegeben sein; wenn sie dies aber nicht sind, dann unterliegen sie dem Wandel, dann können die Privilegierten von heute die Ausgestoßenen von morgen sein“. Die Grundlagen sozialer Ungleichheitsverhältnisse wurden nun in gesellschaftlichen Umständen - etwa der unterschiedlichen Verteilung von Privilegien in der Ständegesellschaft - erblickt. Den ideengeschichtlich prominentesten Ausdruck dieser Idee hat wohl Jean-Jacques Rousseau formuliert. Gleich zu Beginn seines Diskurses Über den Ursprung der Ungleichheit unter den Menschen unterscheidet er zwischen der „,natürlichen“ oder ,physischen“ Ungleichheit der körperlichen Konstitution und Intelligenz der Menschen einerseits und der „moralischen“ oder „politischen“ (sprich: sozialen) Ungleichheit andererseits, die ,,von einer Art Konvention abhängt und durch die Zustimmung der Menschen begründet oder zumindest autorisiert wird“ (Rousseau 1984, S. 67). Die Entstehung dieser Art von Ungleichheit gründet Rousseau zufolge in einem Akt der „Landnahme“ und der Zustimmung hierzu durch den Rest der Gesellschaft (Berger 2004, S. 358; Rousseau 1984, S. 173 ff.).

Damit wird die Wahrnehmung und Erklärung gesellschaftlicher Ungleichheitsverhältnisse - in den Worten von Giesen (1987, S. 315) - „entnaturalisiert“ bzw. genauer: ent-essenzialisiert. Ungleichheit gilt nunmehr als ein Produkt menschlichen Handelns. Als solches ist sie aber zumindest im Grundsatz immer auch veränderbar (Kreckel 2004, S. 13) und daher legitimationsbedürftig: „Wenn nun soziale Ungleichheit dem Bereich des Handelns und Entscheidens unterstellt wird“, betont Giesen (1987, S. 315), „,so ergibt

2 Gleichwohl gab es auch in voraufklärerischen Zeiten Gegenströmungen zu diesen dominanten Perspektiven. Das frühe Christentum etwa postulierte die Gleichheit aller Gläubigen in und durch den Glauben, der auch den Heiden offen stand. 
sich daraus auch die Möglichkeit, sie in Frage zu stellen, und die Notwendigkeit, sie zu rechtfertigen. Anders als natürliche Ungleichheit ist soziale Ungleichheit nicht selbstverständlich, sondern kann zum Gegenstand der Kritik unter Gerechtigkeits- und das heißt vor allem: unter Gleichheitsgesichtspunkten - werden“. Kurz: die Wahrnehmung des gesellschaftlichen Ursprungs sozialer Ungleichheit - und damit ihrer grundsätzlichen Veränderbarkeit - bildet eine wichtige Grundlage für die Entfaltung de-legitimierender, ungleichheitskritischer Sichtweisen auf die gesellschaftlichen Verhältnisse. Entsprechend ist die Idee der Gerechtigkeit an die Ausbildung eines Bewusstseins der „Konstruktivität“ von Gesellschaft geknüpft (vgl. Dux 2008, S. 27, 64 ff.). ${ }^{3}$

Infolge dieses Legitimationsdrucks auf den gesellschaftlichen Status quo müssen den Benachteiligten der herrschenden Gesellschaftsordnung ,gute Gründe“ angeboten werden, diese Ordnung zu akzeptieren. Erst durch die Zustimmung der Betroffenen kann Ungleichheit Legitimität gewinnen und sich verfestigen oder reproduzieren (vgl. Berger 2004, S. 355). In diesem Sinne spricht Kreckel (2004, S. 23) von einem „Vulkan der Interessen der jeweils benachteiligten Bevölkerungsmehrheiten“, dessen erfolgreiche Eindämmung die Voraussetzung für die Fortexistenz sozialer Ungleichheit sei.

Diese Argumentation bildet den aufklärerischen Common Sense der Ungleichheitssoziologie. Obgleich selten explizit thematisiert, tritt er - in teils unterschiedlichen Aspekten - in Einführungen in die Ungleichheitssoziologie deutlicher zutage, so etwa bei Burzan (2004, S. 8 ff.), Barlösius (2005, S. 12), Kreckel (2004, S. 23 ff.), Solga et al. (2009, S. 11) oder Schwinn (2007, S. 41 f.). Doch enthält dieser aufklärerische Common Sense realistische Annahmen über die Ungleichheitsdeutungen der Gesellschaftsmitglieder? ${ }^{4}$ Wird Ungleichheit in modernen Gesellschaften tatsächlich als ein auf gesellschaftliche Umstände rückführbares Produkt menschlichen Handelns wahrgenommen, und ergibt sich hieraus ein besonderer Legitimationsdruck? Um diese Fragen zu beantworten, ist es notwendig, sich der Wahrnehmung sozialer Ungleichheit durch die Gesellschaftsmitglieder zuzuwenden.

3 Die Einsicht, dass Ungleichheitsstrukturen von Menschen geschaffene „soziale Tatsachen“ sind, impliziert lediglich, dass sie grundsätzlich veränderbar sind, nicht aber, wie leicht oder schwierig dies ist. Weder ist damit gesagt, dass Ungleichheitsverhältnisse nach Belieben zur Disposition stünden, noch, dass sie eine intendierte Handlungsfolge sind (vgl. Kreckel 2004, S. 14). Im Gegenteil sind Ungleichheitsverhältnisse - gerade als soziale Tatsachen - oftmals von großer Persistenz und Hartnäckigkeit, wie etwa die Geschlechterforschung zeigt (Gottschall 2000).

4 Man könnte an dieser Stelle einwenden, dass die postulierten Veränderungen in der Wahrnehmung gesellschaftlicher Ungleichheitsverhältnisse sich nicht auf die Wahrnehmungen breiter Bevölkerungskreise erstreckten, sondern allenfalls auf gebildete Kreise. Selbst wenn dieses Argument zutreffen sollte, ist die - möglicherweise durch eine solche Elite gesteuerte - Verbreitung dieser Wahrnehmung in großen Bevölkerungskreisen notwendig, um einen hinreichenden Legitimationsdruck zu entfalten. 


\section{Deutungsmuster sozialer Ungleichheit}

Ein Blick auf quantitative Umfragedaten zeigt zunächst, dass eine Mehrheit der deutschen Bevölkerung soziale Umstände als Gründe für soziale Ungleichheit anführt und sich ihre Wahrnehmungen im Einklang mit den Annahmen des aufklärerischen Common Sense zu befinden scheinen. So zeigen ALLBUS-Daten aus dem Jahr 2000, dass $75 \%$ der deutschen Bevölkerung der Aussage zustimmen, die sozialen Unterschiede blieben bestehen, „weil sie den Reichen und Mächtigen nützen“ (eigene Berechnungen, vgl. Tab. 2 im Anhang). Im Jahr 2004 waren überdies knapp $58 \%$ der Auffassung, es gebe in Deutschland noch große Unterschiede zwischen den einzelnen Schichten, und was man im Leben erreiche, hänge im Wesentlichen vom Elternhaus ab. Diese Wahrnehmungen indizieren ein Verständnis von Ungleichheit als durch gesellschaftliche Faktoren verursacht. Dennoch sind sie nicht frei von Ambivalenzen und Widersprüchlichkeiten. Denn zugleich ist eine große Mehrheit der Befragten (etwa 79 \%) der Auffassung, dass Deutschland eine offene Gesellschaft sei, und was man erreiche, hänge von der Bildung und den eigenen Fähigkeiten ab (vgl. Tab. 2 im Anhang).

Derartige Widersprüchlichkeiten sind in der einschlägigen Forschungsliteratur nicht unbekannt (vgl. u. a. Kluegel und Smith 1986; Mayer et al. 1992; Svallfors 2006). Ein aktueller Überblick über die Forschungsliteratur in Großbritannien gelangt zu dem Schluss, dass die Befunde der quantitativen Umfrageforschung zur Wahrnehmung sozialer Ungleichheit ,complex, ambiguous and contradictory“ (Orton und Rowlingson 2007, S. 40) seien. Diese Ambivalenzen lassen sich im Rahmen quantitativer Umfrageforschung nicht auflösen. Um ihnen nachzuspüren und aufzudecken, wie (logisch) widersprüchliche Ungleichheitswahrnehmungen von den Befragten praktisch in Einklang gebracht werden, erscheint eine qualitative Herangehensweise aussichtsreich. „Future research“, so fordern Orton und Rowlingson (ebd., S. 42), ,also needs to focus more on people's underlying values, the discourses they draw on and how they understand concepts such as inequality“. In diesem Sinne wird hier ein qualitativer Zugang zur Wahrnehmung sozialer Ungleichheit gewählt.

Unter ,sozialer Ungleichheit“ werden im Anschluss an Kreckel (2004, S. 17) die relativ dauerhaften Muster von Begünstigung und Benachteiligung verstanden, die bestimmte Individuen oder Gruppen im Zugang zu begehrten Gütern oder Positionen erfahren. Was die begehrten Güter oder Positionen im Einzelnen sind und welche spezifischen Gruppen als begünstigt oder benachteiligt gelten, wird im Sinne des qualitativen Forschungsdesigns bewusst offengehalten, denn die Relevanz einzelner Dimensionen oder Erscheinungsformen sozialer Ungleichheit ist letztlich nur normativ begründbar (hierzu instruktiv Schmidt 2004). Soziologisch lässt sich bestimmen, welche Erscheinungsformen sozialer Ungleichheit den Betroffenen selbst als problematisch (und daher „relevant“) erscheinen (Mau 2004). Diese Herangehensweise wird hier gewählt.

Grundlegend ist dabei die wissenssoziologische Prämisse, dass die individuelle Wahrnehmung und Erfahrung der Menschen auf sozial geteilte Wissensvorräte und Deutungsschemata angewiesen ist, um die Realität angemessen zu interpretieren (Schütz 1964, S. 231; 1974). Für die Wahrnehmung sozialer Ungleichheit verdeutlicht dies insbesondere Katherine Newmans Untersuchung zur subjektiven Verarbeitung sozialer Abwärtsmobilität in den USA (Newman 1999). Soziale Abstiegsprozesse sind für die Betroffenen nicht 
nur aufgrund der mit ihnen einhergehenden materiellen Einschränkungen problematisch, sondern auch, weil ihnen entsprechende kulturelle Schablonen zur sinnhaften Verarbeitung dieser Erfahrungen fehlen. Sie können daher oftmals kaum umhin, ihren Abstieg als individuelles Versagen zu interpretieren, auch wenn es sich um strukturell bedingte Phänomene handelt. „Downward mobility“, so Newman (ebd., S. 9), ,has virtually no ritual face. It is not captured in myths or ceremonies that might help individuals in its grip to make the transition from a higher to a lower social status [...]. They mourn in isolation and fail to reach any sense of closure in their quest for a new identity. Their disorientation suggests how critical culture is in ,explaining to individuals the meaning of their fate“". Die kulturell verfügbaren Deutungen von Ungleichheit spielen demnach für die sinnhafte Verarbeitung individueller Erfahrungen eine wichtige Rolle.

Diese Einbettung individueller Sichtweisen in sozial geteilte Interpretationsfolien wird im Konzept des „,sozialen Deutungsmusters“ (Meuser und Sackmann 1992; Oevermann 2001c; Ullrich 1999) theoretisch gefasst. Unter einem sozialen Deutungsmuster wird ein von mehreren Individuen geteiltes Interpretationsmuster verstanden, das mit einem bestimmten Bezugsproblem (hier: der Wahrnehmung sozialer Ungleichheit) verbunden ist, normative Geltungskraft besitzt und den Akteuren nur in Grenzen reflexiv verfügbar ist (vgl. Oevermann 2001a, 2001c; Ullrich 1999). Als kollektive Interpretations- und Legitimationsangebote sind Deutungsmuster konstitutiv für individuelle Handlungsorientierungen und Situationsdefinitionen (Oevermann 2001c, S. 19; Ullrich 1999, S. 429). Empirisch manifestieren sie sich jedoch immer nur in spezifisch individuellen Ausprägungen und Anwendungen, weshalb sie nicht direkt erhoben, sondern nur aus Vergleichen individueller Äußerungen und Begründungen rekonstruiert werden können (vgl. Abschn. 4).

Theoretisch kann erwartet werden, dass sich Ungleichheitsdeutungen zwischen den Mitgliedern verschiedener sozialer Gruppen (z. B. Klassen oder Schichten) unterscheiden. Deutungsmuster werden als „,naturwüchsig aus einer Problemstellung einer historisch spezifischen Lage hervorgegangene ,Weltbilder' [...], als Gebilde also, die integral eine Milieu- oder Subkulturzugehörigkeit konstituieren“ (Oevermann 2001b, S. 541), verstanden. Insofern sich also die gesellschaftlichen Verhältnisse, in denen Menschen leben, unterscheiden, benötigen sie auch unterschiedliche Deutungsmuster, um diese Verhältnisse zu interpretieren und sinnhaft zu strukturieren. Daher sollten sich im Gruppenvergleich verschiedene, um eine verbindliche Wirklichkeitsdeutung konkurrierende Deutungsmuster sozialer Ungleichheit finden (Berger 1988, S. 508).

Bei Deutungsmustern sozialer Ungleichheit ist es zudem wichtig, zwischen ihrer kognitiven und normativen Dimension zu unterscheiden, also zwischen der kognitiven Repräsentation sozialer Ungleichheit in den Köpfen der Menschen einerseits sowie ihrer normativen Sicht darauf andererseits (Mau 2004, S. 186). Auf der kognitiven Ebene geht es somit um die „Topologie“ (Berger 1988, S. 508) sozialer Ungleichheit - d. h. die mentale Landkarte, mittels derer die Menschen die gesellschaftliche Ungleichheitsstruktur wahrnehmen und sich in ihr verorten - sowie um die Erklärungen und Ursachenattributionen für die wahrgenommenen Ungleichheiten. Auf der normativen Ebene der Ungleichheitsdeutung hingegen geht es um die Bewertung und Legitimation sozialer Ungleichheit durch die Befragten, d. h. ihre normativen Orientierungen hinsichtlich bestehender Ungleichheitsverhältnisse. Die wohl am besten erforschte Form derartiger 
„Begründungsordnungen“ (Boltanski und Thévenot 1999) sozialer Ungleichheit sind Gerechtigkeitsorientierungen (Hochschild 1981; Miller 1999; Wegener 1992), obgleich sich Rechtfertigungen sozialer Ungleichheit aber auch auf externe Sachzwänge o. Ä. stützen können (Nollmann 2003; Schmidt 2000).

Diese Differenzierung ist zunächst rein analytischer Natur, denn empirisch können einzelne Deutungsmuster kognitive und normative Elemente zugleich (auch zu je unterschiedlichen Anteilen) enthalten. Die hier behandelten Deutungsmuster der Ursachen sozialer Ungleichheit beziehen sich auf die kognitive Ebene der Ungleichheitsdeutung. Im Sinne des aufklärerischen Hintergrundkonsenses wäre jedoch zu erwarten, dass bestimmte Ursachenattributionen auch spezifische normative Haltungen der Befragten nahelegen - etwa, dass Deutungsmuster, die Ungleichheit als soziales Konstrukt betrachten, mit ungleichheitskritischen Haltungen einhergehen. Um derartige Implikationen bestimmter kognitiver Deutungen für die normativen Orientierungen der Befragten herauszuarbeiten, ist bei der empirischen Rekonstruktion zwischen der kognitiven und normativen Dimension zu differenzieren (Mau 2004, S. 186).

\section{Datengrundlage und Auswertungsmethode}

Die Datengrundlage bilden 20 leitfadengestützte diskursive Interviews (Ullrich 1999) mit Angehörigen privilegierter und benachteiligter sozialstruktureller Lagen. Beim diskursiven Interview handelt es sich um eine eigens für die Rekonstruktion sozialer Deutungsmuster entwickelte Form des leitfadengestützten Interviews. Da soziale Deutungsmuster empirisch nur über die Analyse ihrer individuellen Anwendungen (,Derivationen“, vgl. ebd.) zugänglich sind, kommt es in der Befragungssituation auf die Generierung von solchem Daten- bzw. Textmaterial an, anhand dessen sich die den Einzelaussagen zugrunde liegenden Deutungsmuster über eine verdichtende und typisierende Analyse rekonstruieren lassen. Zu diesem Zweck sieht das diskursive Interview - im Unterschied zu traditionellen, eher zurückhaltenden Interviewformen - eine vergleichsweise starke Strukturierung des Leitfadens sowie den Einsatz expliziter steuernder Eingriffe seitens des Interviewers vor. Die Strukturierung des Leitfadens gewährleistet dabei ein Mindestmaß an Vergleichbarkeit und Konstanz der behandelten Themen über die einzelnen Interviews hinweg, wobei in der Befragungssituation eine „Leitfadenbürokratie“ (Hopf 1978) natürlich vermieden werden sollte. Die Interviewführung beinhaltet insbesondere solche Frageformen, welche die Befragten zu Stellungnahmen zu bestimmten Sachverhalten und den entsprechenden Begründungen dieser Ansichten veranlassen sollen, da diese Textsorten für die Rekonstruktion sozialer Deutungsmuster wesentlich sind (vgl. ausführlich Ullrich 1999).

Die Interviews wurden von April bis September 2007 im Raum Bremen geführt. Über ein Schneeball-Verfahren wurden gezielt Befragte aus privilegierten und benachteiligten sozialstrukturellen Lagen rekrutiert. Das zentrale Auswahlkriterium war die Klassenlage der Befragten in Anlehnung an das EGP-Klassenschema (Erikson und Goldthorpe 1992). Als Befragte aus den privilegierten Lagen wurden sechs Angehörige der oberen Dienstklasse sowie vier Selbstständige (Akademiker) ausgewählt (Interviews B-1 bis B-6 und D-1 bis D-4). Die Angehörigen benachteiligter Lagen umfassen zwei Facharbeiter, vier 
einfache Angestellte mit nicht-manuellen Routinetätigkeiten (davon zwei ALG-II-Aufstockerinnen), drei Langzeitarbeitslose sowie eine Hausfrau (Interviews A-1 bis A-4 und C-1 bis C-6). In beiden Gruppen wurden zu etwa gleichen Anteilen Männer und Frauen rekrutiert. ${ }^{5}$ Die nicht in den Arbeitsmarkt integrierten Personen (Arbeitslose, eine Hausfrau) wurden einbezogen, um den „Erwerbstätigenbias“ vieler Klassenschemata zu überwinden. Sie wurden unter Berücksichtigung ihres vorherigen Berufs (bzw. im Fall der Hausfrau des Berufs des Ehemanns) den benachteiligten Klassenlagen zugerechnet.

Die Interviews dauerten im Schnitt zwischen 60 und 120 Minuten. Darin wurden Wahrnehmungen zu Ausmaß, Formen, Ursachen, Rechtfertigung und Folgen sozialer Ungleichheit in Deutschland thematisiert. Zu Beginn eines Interviews wurde gefragt, ob die Interviewpartner einen Anstieg sozialer Ungleichheit in Deutschland wahrnehmen, und falls ja, woran sie diesen festmachen bzw. „sehen“ können. Neben der allgemeinen Einführung in das Interviewthema diente diese Frage auch dazu, das subjektive Verständnis der Befragten von ,sozialer Ungleichheit“ zu erschließen. Im Anschluss daran sollten die Interviewpartner angeben, welche sozialen Gruppen in ihren Augen zu den Benachteiligten und den Begünstigten des gegenwärtigen Ungleichheitsgefüges in Deutschland gehören, wie sie ihre eigene Lage darin beschreiben würden und was ihrer Ansicht nach die Ursachen sozialer Ungleichheit seien. Schließlich wurde nach ihren normativen Orientierungen, ihrer Interpretation verschiedener Gerechtigkeitsorientierungen und den wahrgenommenen (individuellen wie gesellschaftlichen) Konsequenzen sozialer Ungleichheit gefragt. Die folgenden empirischen Befunde stützen sich vor allem auf die Interviewsegmente zu den wahrgenommenen Ursachen und den normativen Orientierungen. Im Anschluss an das Interview wurden die Interviewpartner gebeten, einen standardisierten Kurzfragebogen zu verschiedenen soziodemografischen Merkmalen auszufüllen, die als Kontextinformation für die Analyse dienten.

Alle Interviews wurden vollständig transkribiert und sowohl induktiv als auch deduktiv inhaltlich kodiert. Bei der Auswertung wurde in einem ersten Schritt eine thematische Kodierung aller Interviewtranskripte in Anlehnung an die im Leitfaden enthaltenen Themen vorgenommen. Die so entstandenen Kategorisierungen wurden in einem zweiten Schritt anhand einer synoptischen Lektüre des gesamten Textmaterials zu einer spezifischen Kategorie verfeinert (Bildung von Subkategorien) und um neue Kategorien erweitert, sodass hier von einer Mischung aus deduktivem und induktivem Vorgehen gesprochen werden kann (Kelle und Kluge 1999; Kuckartz 2006). Im Rahmen solcher synoptischen Vergleiche der Äußerungen und Ansichten aller Interviewpartner zu einem

5 Die Auswahl dieser konkreten Gruppen basiert auf der Überlegung, dass Personen an den beiden „Extrempolen“ des Ungleichheitsspektrums ein größeres Bedürfnis nach Erklärung und Rechtfertigung (bzw. Kritik) der eigenen sozialstrukturellen Lage (bzw. derjenigen anderer) unterstellt werden kann als den Angehörigen sozialstruktureller Mittellagen. Empirisch zeigen quantitative Analysen des ALLBUS 2004, dass Facharbeiter, un-/angelernte Arbeiter, nichtmanuelle Angestellte mit niedriger Qualifikation (einfache Angestellte) und Arbeitslose mit höherer Wahrscheinlichkeit angeben, weniger als ihren ,gerechten Anteil am Lebensstandard“" zu erhalten als Angehörige der oberen und unteren Dienstklasse, nicht-manuelle qualifizierte Routineangestellte sowie Selbstständige. Diese Unterschiede bestehen auch unter Kontrolle verschiedener soziodemografischer Merkmale (Daten hier aus Platzgründen nicht ausgewiesen, vgl. Sachweh 2010, S. 325). 
bestimmten Bezugsproblem (hier: den wahrgenommenen Ursachen sozialer Ungleichheit) treten die Gemeinsamkeiten und Unterschiede zwischen den einzelnen Deutungen immer deutlicher hervor. Das Ergebnis dieser vergleichenden und die einzelnen (Sub-) Kategorien sukzessive verdichtenden Analyse ist die Rekonstruktion unterschiedlicher, empirisch abgesicherter Deutungsmuster (Ullrich 1999).

\section{Unvermeidbarkeit und Herkunftsbedingtheit - Alltagsweltliche Erklärungsmuster sozialer Ungleichheit}

Der empirische Teil dieses Artikels fragt, inwieweit die im aufklärerischen Common Sense enthaltenen Annahmen über das Alltagswissen der Akteure realistisch sind. Dazu wird untersucht, wie Ungleichheit von Menschen in privilegierten und benachteiligten sozialstrukturellen Lagen erklärt wird. Es werden zwei Deutungsmuster von Ungleichheitsgründen rekonstruiert, die sich darin unterscheiden, inwieweit sie soziale Ungleichheit als ein von Menschen gemachtes Konstrukt oder als notwendigen Ausdruck gesellschaftlicher Ordnung betrachten (ähnlich Terpe 2009, S. 102) (vgl. Tab. 1). ${ }^{6}$

Zudem wird gefragt, welche Implikationen sich aus diesen Deutungsmustern für die normativen Orientierungen der Befragten ableiten lassen. Gehen mit Deutungsmustern, die Ungleichheit als gesellschaftlich bedingt erachten, ungleichheitskritischere normative Orientierungen aufseiten der Interviewpartner einher als mit Deutungsmustern, die Ungleichheit als unvermeidbare Notwendigkeit gesellschaftlicher Ordnung betrachten?

5.1 „Natürlich ist das nicht gerecht, das ist tierisch ungerecht. Aber es ist ganz einfach so“ - Deutungsmuster der Unvermeidbarkeit sozialer Ungleichheit

Die Ansicht, Ungleichheit sei ein unvermeidbarer Bestandteil einer jeden gesellschaftlichen Ordnung, ist ein immer wiederkehrendes Thema in den Interviews. Die Begründungen dieser Unvermeidbarkeit reichen dabei von Verweisen auf die bloße Faktizität von Ungleichheit über ihre historisch-traditionale Erklärung bis hin zu Deutungen, die soziale Ungleichheit mit der menschlichen Natur im Allgemeinen in Verbindung bringen. ${ }^{7}$ Es

6 In der Literatur wird bezüglich der subjektiven Erklärungsmuster von Ungleichheit meist zwischen individuellen und strukturellen/sozialen Ursachen unterschieden. Im ersten Fall werden Ungleichheiten in den individuellen Eigenschaften der Akteure selbst (z. B. Bildung, Leistungsbereitschaft) verortet, im zweiten Fall werden sie als strukturell oder sozial bedingt angesehen (vgl. Feagin 1975; van Oorschot und Halman 2000; Will 1993). Darüber hinaus hat bereits Feagin (1975) auf „fatalistische“ Sichtweisen hingewiesen, welche die Ursache beispielsweise für Armut in überindividuellen, nicht-sozialen Ursachen wie z. B. Pech oder Schicksal sehen. Diese Unterscheidungen stellen allerdings analytische Abstraktionen dar, die von den Befragten so nicht geteilt werden.

7 Dabei bezieht sich die Unvermeidbarkeitsdeutung keineswegs ausschließlich auf soziale Ungleichheit als allgemeines, mithin ,,ahistorisches“ Phänomen (im Sinne einer Universalität von Privilegierung und Benachteiligung). Vielmehr wird sie, wie noch deutlich wird, auch zur Erklärung konkreter Ungleichheitslagen angewandt, etwa bei der Betonung von Persönlichkeitseigenschaften für die Überwindung von Arbeitslosigkeit. 
Tab. 1: Deutungsmuster von Ungleichheitsgründen

\begin{tabular}{|c|c|}
\hline Unvermeidbarkeit sozialer Ungleichheit & Herkunftsbedingtheit sozialer Ungleichheit \\
\hline $\begin{array}{l}\text { - Ungleichheit als Tatsache } \\
\text { - Ungleichheit als historische Kontinuität } \\
\text { - Ungleichheit aufgrund verschiedenartiger } \\
\text { Anlagen und Fertigkeiten } \\
\text { - Ungleichheit als Teil der menschlichen } \\
\text { Natur }\end{array}$ & $\begin{array}{l}\text { - materielle Bedingungen der sozialen Herkunft } \\
\text { (direkt/indirekt) } \\
\text { - soziokulturelle Einflüsse der sozialen Herkunft }\end{array}$ \\
\hline Notwendigkeit & soziales Konstrukt \\
\hline
\end{tabular}

lässt sich damit eine Fortexistenz voraufklärerischer bzw. essenzialistischer Ungleichheitsdeutungen festhalten, die nicht mit den Annahmen des aufklärerischen Hintergrundkonsenses der Ungleichheitssoziologie in Einklang stehen. Das Deutungsmuster der Unvermeidbarkeit sozialer Ungleichheit tritt in vier verschiedenen Varianten auf, die sich ihrer Komplexität nach ordnen lassen:

1) In seiner einfachsten Form bezieht es sich auf die bloße Faktizität sozialer Ungleichheit. Die ungleiche Verteilung begehrter Güter und Positionen wird hier schlicht als Tatsache gesehen, die einen zwar bedauerlichen, aber eben doch vorhandenen Bestandteil der gesellschaftlichen Wirklichkeit darstellt, der sich auch künftig nicht (oder zumindest nicht wesentlich) verändern oder verringern lasse. Die gegenwärtige Faktizität sozialer Ungleichheit wird so in die Zukunft extrapoliert. Bisweilen wird der Realität sozialer Ungleichheit auch ein Ideal von Gleichheit gegenübergestellt, das sich in den Augen der Befragten als unrealisierbar erweist. So meint ein promovierter Betriebswirt:

„Es wäre schön, wenn alle Menschen gleich wären und jeder hätte den gleichen Standard, aber es ist einfach nicht so. Ich versuch' das gar nicht so zu werten, sondern ich seh' das einfach als Tatsache. Natürlich, wenn man richtig in sich reinguckt und denkt, ,Mensch, es ist richtig so, dass jemand, [...] der als Sohn von einem Herrn Piech geboren wird, dass der eine ganz andere Startmöglichkeit als wenn jemand hier in Gröpelingen [...] von einer alleinerziehenden Mutter geboren wird, die Sozialhilfeempfängerin ist". Natürlich ist das nicht gerecht, das ist, das ist tierisch ungerecht. Aber es ist ganz einfach so." (Interview B-1) ${ }^{8}$

Die Implikationen dieses Deutungsmusters für die normativen Orientierungen der Befragten klingen im letzten Satz dieses Zitates bereits an (,Aber es ist ganz einfach so.“). Sie werden auch in der Aussage einer 46-jährigen Hausfrau deutlich, die auf die Frage, was man ihrer Ansicht nach gegen soziale Ungleichheit unternehmen könne, entgegnet: „Dagegen kann man ja eigentlich nix machen. Das ist der Welten Lauf, 'ne“ (Interview C-4). Obwohl die Unvermeidbarkeitsdeutung kein explizites Legitimationsmuster darstellt, scheint hier eine gewisse „normative Kraft des Faktischen“ insoweit wirksam zu sein, als den Befragten eine Gesellschaft ohne oder mit weniger Ungleichheit nur schwer vorstellbar erscheint.

8 In Klammern nach dem Zitat ist die anonymisierte Kennnummer des Interviewtranskripts angegeben. Längere Sprechpausen werden mit „...“ gekennzeichnet, Auslassungen aus Zitaten mit ,[...]“. Die Zitate wurden zur besseren Lesbarkeit editiert. 
2) Eine weitere Variante der Unvermeidbarkeitsdeutung thematisiert die historische Kontinuität sozialer Ungleichheit. Soziale Ungleichheit, so der Grundgedanke, habe es schon immer gegeben und werde es daher auch weiterhin geben. So verweisen einige Befragte darauf, dass es bereits in früheren Zeiten „Reiche und Mittlere und Arme“ gegeben habe und sich dies nun fortsetze. Das Argument, mit dem hier Ungleichheit „erklärt“ wird, stützt sich auf ihre Existenz über einen längeren historischen Zeitraum hinweg. So sieht dies beispielsweise eine ungelernte Angestellte:

„Weil der Ursprung selber von dem ganzen Kram liegt ja schon Millionen Jahre zurück, so ungefähr, nich'. Wieso hat einer 'n Haus und hat Land ohne Ende und der andere hat gar nichts. Das liegt ja schon, na ja, kann man ja schon gar nicht mehr denken, wo das schon zurückliegt. [...] Der eine hat 500 Hektar Land und der andere hat gar nichts, der ist 'n Bauer ohne Magd. Ja, wie kommt das, wo kommt das her? Das ist doch schon immer so gewesen, kannst Du praktisch auf gut Deutsch sagen." (Interview C-5)

Diese Ansicht wird auch von anderen Befragten geteilt. So meint etwa eine Postzustellerin, dass es Ungleichheit ,ja schon immer gegeben [hat], das wird's ja auch immer [geben]" (Interview C-2). Da die gegenwärtige Existenz von Ungleichheit als Fortsetzung schon immer bestehender Verhältnisse von Privilegierung und Benachteiligung betrachtet wird, scheint für diese Befragten auch in Zukunft wenig Aussicht auf Veränderung zu bestehen. Die wahrgenommene Kontinuität untermauert nur die Hartnäckigkeit und Universalität sozialer Ungleichheit und nährt zugleich Zweifel an ihrer prinzipiellen Veränderbarkeit. Dennoch bleiben sowohl der Verweis auf die Faktizität als auch die historische Kontinuität sozialer Ungleichheit als Erklärung unbefriedigend - sowohl analytisch als auch aus Sicht der Befragten. Daher belässt es kaum ein Interviewpartner bei diesen Hinweisen.

3) In einer etwas komplexeren Variante der Unvermeidbarkeitsdeutung verweisen die Befragten auf die Verschiedenheit der Menschen in ihren Anlagen und Fähigkeiten, um die zwischen ihnen bestehenden Ungleichheiten zu erklären. Dabei legen sie die Vorstellung zugrunde, dass die Unterschiede in den individuellen Anlagen und Fähigkeiten der Menschen, z. B. in der Intelligenz oder der Auffassungsgabe, ihre Position im Ungleichheitsgefüge beeinflussen (vgl. Lamont 2000, S. 219 ff.). Ungleichheit wird hierdurch - entgegen der Annahmen des aufklärerischen Common Sense - „(re-)naturalisiert“. Aus der Verschiedenheit der natürlichen Anlagen der Menschen folgt aus dieser Sicht zwangsläufig, dass jene mit weniger, ,vorteilhaften“ Anlagen zurückbleiben und es so zu einer Art negativer Auslese kommt. Diese Auffassung kommt deutlich in der folgenden Äußerung eines 70-jährigen Unternehmers im Ruhestand zum Ausdruck, als er im Laufe des Interviews über die Gründe für Arbeitslosigkeit und seine eigenen Erfahrungen in Bewerbungsgesprächen mit Arbeitssuchenden spricht: „Dann gibt's auch andere Gründe, einfach im Wissen. Wenn man die also testet und stellt fest, der eine ist intelligenter und fasst schneller auf, dann nimmt man den. Da erfolgt dann ja wieder eine Auslese, und so entsteht auch eine Auslese negativer Art, das heißt ein Häuflein, die schaffen's einfach nicht, warum auch immer. Die gibt's auch.“ (Interview B-6) In den Augen dieses Interviewpartners führen Unterschiede der Menschen in ihren Anlagen und Fähigkeiten zur Herausbildung eines „Rests“ von in diesen Anlagen am wenigsten begünstigten Personen (,ein Häuflein, die schaffen's einfach nicht“) - und somit auch zu Ungleichheit. 
Darüber hinaus verweisen die Befragten neben allgemeinen kognitiven Fähigkeiten auch auf bestimmte Persönlichkeitseigenschaften, die für die Positionierung im Ungleichheitsgefüge relevant seien. So werden denjenigen, die benachteiligte Positionen in der Sozialstruktur besetzen, ein zu geringes Aspirationsniveau, alternative Wertsetzungen oder Erfolgsdefinitionen (in Form eines ,value stretch“, vgl. Hyman 1966; Rodman 1963), Antriebslosigkeit, Introvertiertheit, Labilität, fehlende Belastbarkeit oder ein Mangel an Hartnäckigkeit zugeschrieben. Der bereits zitierte 30-jährige Betriebswirt erblickt etwa in einem niedrigen Aspirationsniveau eine Ungleichheitsursache. Er findet, „es gibt auch manche Leute, die sagen: ,Ich will da auch gar nicht nach oben [...]. Ich bin hier in dieser Schicht, und da gehör ich hin!' [...] Menschen sind einfach verschieden, und dadurch wird es auch soziale Ungleichheit weiterhin geben“ (Interview B-1). Ähnlich sieht dies ein Unternehmer, der der Ansicht ist, dass „,der größte Fehler ist, sich mit wenig zufrieden zu geben. Das heißt, ich habe hier eine Arbeit, ich fange morgens um 8 an, ich gehe um 17 Uhr nach Hause, ich kriege dafür ein Gehalt, das reicht grade aus, um mich und meine Familie zu ernähren, und das war's, ne? Wer sich damit zufrieden gibt, muss sich natürlich nicht wundern, wenn dieser Zustand auch permanent bleibt durch fast sein ganzes Leben." (Interview B-6)

In eine ähnliche Richtung geht die Auffassung einer Personalberaterin, die glaubt ,dass einige weniger mitbekommen, einfach von ihrer Persönlichkeit her, und andere mehr. Und das wird sich immer in der Gesellschaft darstellen“ (Interview D-2). Diese Beispiele verdeutlichen, wie die Interviewpartner eine Verbindung zwischen bestimmten Persönlichkeitseigenschaften und der Stellung in der Ungleichheitsstruktur herstellen. Insbesondere niedrige Aspirationen und geringe Ambitionen sind in ihren Augen mitverantwortlich für eine benachteiligte Stellung in der Gesellschaft. ${ }^{9}$ Solche Persönlichkeitseigenschaften werden von den Interviewpartnern als rein individuelle Charaktereigenschaften angesehen. ${ }^{10}$ So meint ein 38-jähriger Unternehmer, als er über die Schwierigkeiten von Arbeitslosen, die wiederholt mit der Erfolglosigkeit von Bewerbungen umgehen müssten, spricht:

„Der eine hat mehr Selbstdisziplin, sagt: ,Komm, es geht weiter, und immer einmal mehr aufstehen als hinfallen'. Und, aber der andere sagt, ,Jetzt hab' ich genug Bewerbungen geschrieben, das ist eh zwecklos.' Das sind natürlich individuelle Merkmale eines jeden, die nicht unbedingt auf, auf den sozialen oder den gebildeten Grad schließen lassen. Das sind, denk' ich, Charaktereigenschaften, der eine ist disziplinierter, der andere nicht [...].“ (Interview B-5)

Hier wird deutlich, dass bestimmte Eigenschaften (wie z. B. Disziplin) als wichtig für ein erfolgreiches Berufsleben angesehen werden. Somit schreibt diese Variante der Unvermeidbarkeitsdeutung die Verantwortung für Privilegierung und Benachteiligung den Individuen zu und trägt darüber indirekt zur Legitimation sozialer Ungleichheit bei.

9 Denjenigen dagegen, die eine privilegierte Position in der Sozialstruktur besetzen, werden entsprechend positive persönliche Eigenschaften (wie z. B. Zielstrebigkeit, Fleiß, Leistungsbereitschaft, Beharrlichkeit, Flexibilität, Disziplin, Durchsetzungsvermögen) zugeschrieben, die als Grundlage für die erreichte Position wahrgenommen werden.

10 Dies steht im Widerspruch zu Befunden, die belegen, dass Persönlichkeitseigenschaften auch von der sozialstrukturellen Lage beeinflusst werden (vgl. u. a. Kohn 1977, 1980). 
4) Die Unvermeidbarkeit sozialer Ungleichheit wird schließlich auch mit dem Verweis auf die menschliche Natur begründet. Hier unterstellen die Interviewpartner, das Wesen des Menschen sei durch anthropologisch konstante Eigenschaften wie Neid, Ehrgeiz, Egoismus, Individualismus, Erfolgsstreben und Raffgier bestimmt, die mit Bestrebungen nach größerer sozialer Gleichheit nicht vereinbar seien. Es sind also nicht nur individuelle Unterschiede in den Fähigkeiten und Persönlichkeitseigenschaften, die für die Unvermeidbarkeit sozialer Ungleichheit verantwortlich gemacht werden, sondern auch als universal angesehene Merkmale der menschlichen Natur. Teils verweisen sie in diesem Zusammenhang auch auf ehemals kommunistische Gesellschaften, die ihren egalitären Ansprüchen nicht zuletzt aufgrund dieser (vermeintlich),,anthropologischen“ Grundkonstanten nicht hätten gerecht werden können. Typisch sind in diesem Zusammenhang die folgenden Äußerungen:

„Aber dass alle nun Knäckebrot essen müssen, sag’ ich mal, um’s gleich zu machen, das denke ich, sollte nicht sein. Das wird auch nicht funktionieren. Das heißt, die DDR, wo's versucht wurde, oder Kommunismus, da fuhren alle 'n gleiches Auto und andere fuhren wieder 'n Mercedes im Staat. [...] Ich denke, das liegt leider in der Natur des Menschen, dass das nicht geht. I: Welche Eigenschaften in der Natur des Menschen verhindern das denn? Neid, Ehrgeiz, Profilierungsstreben, einfach, ja, solche Geschichten. Die wenigsten Menschen, denk' ich, sind mit dem zufrieden was sie haben, egal wie viel sie haben.“ (Interview B-5)

„Es war ja mal in der Deutschen Demokratischen Demokratie angedacht, wenn alle das Gleiche haben, sind alle zufrieden. Hatte ja auch nicht funktioniert, nich'. Selbst wenn sie nach 18 Jahren mal ihren Trabbi hatten, wollte der eine ja zumindestens paar Plaste-Radkappen haben, wenn der andere vielleicht noch seine Stahlfelgen hatte. [...] Aber das, das kriegt man ja nicht hin, allen Leuten das Gleiche zu geben. Die werden niemals zufrieden sein." (Interview A-3)

Diese Zitate verdeutlichen sehr klar die Vorstellung der Interviewpartner, dass Menschen gleichsam ,von Natur aus“ danach streben, mehr zu besitzen als andere, und sich mit dem, was sie haben, kaum begnügen würden. Das Potenzial für die Schaffung größerer sozialer Gleichheit werde somit durch diesen universellen Grundzug der menschlichen Natur begrenzt.

Der gemeinsame Kern dieser vier unterschiedlich komplexen Varianten der Unvermeidbarkeitsdeutung besteht darin, dass sie Ungleichheit als unabänderliche, mit der menschlichen Existenz eng verbundene Tatsache und damit als eine unausweichliche Begleiterscheinung gesellschaftlicher Ordnung ansehen. ${ }^{11}$ Welche Konsequenzen ergeben sich nun aus diesem Deutungsmuster für die normativen Orientierungen der Befragten - und damit für die Rechtfertigung sozialer Ungleichheit? Durch die Interpretation von

11 Das Deutungsmuster der Unvermeidbarkeit sozialer Ungleichheit unterscheidet sich in einem wichtigen Punkt von sogenannten ,fatalistischen“ Orientierungen, wie sie neben Feagin (1975) auch die empirische Gerechtigkeitsforschung nachgewiesen hat (Wegener und Liebig 1993). Während fatalistische Sichtweisen auf dem Einzelnen nicht zugängliche, externe Einflüsse und Kräfte - das Schicksal - verweisen, sind es in der Unvermeidbarkeitsdeutung dagegen dem Menschen (bzw. der menschlichen Natur) inhärente Merkmale, die für die Unabänderlichkeit sozialer Ungleichheit angeführt werden. 
Ungleichheit als universellem und notwendigem Bestandteil aller menschlichen Gesellschaften wird bereits auf der kognitiven Ebene der Ungleichheitsdeutung ein zentrales Moment außer Kraft gesetzt, dem im Rahmen des aufklärerischen Common Sense eine tragende Rolle bei der De-Legitimation von Ungleichheitsverhältnissen zugeschrieben wird: die Wahrnehmung der Konstruiertheit gesellschaftlicher Strukturen (Moore 1982). Damit entlastet es die Befragten von einem Rechtfertigungsdruck, der virulenter wäre, wenn Ungleichheit als gesellschaftlich bedingt gälte (vgl. auch Terpe 2009).

Manche Interviewpartner sind sich dieser Implikationen bewusst. So antwortet eine selbstständige Grafikerin auf die Nachfrage, ob sie denn Ungleichheit für unvermeidlich halte: „Ich glaube ja, aber ich scheu mich ein bisschen davor, das zu sagen, weil das ganz böse ausgenutzt werden kann. [I: Warum?] Weil man das gleich abtun kann und sagen kann: ,Ja, und dann ist es doch so, und dann bleibt es so, und gut ist'. Und ich glaube, [...] dann stellt sich dann ja auch immer die Frage nach Gerechtigkeit [...]. Ich glaube, dass es gut ist, 'n Ideal anzustreben, aber letztendlich ist es, glaube ich, 'n Ideal, soziale Gleichheit." (Interview D-4)

Hier werden deutlich die Grenzen artikuliert, die sich durch die Unvermeidbarkeitsdeutung für die normativen Orientierungen der Befragten zu ergeben scheinen. Obgleich ihr die Implikationen der Ansicht, dass sich Ungleichheit faktisch nie beseitigen lassen werde, durchaus bewusst sind und sie es daher befürwortet, „'n Ideal anzustreben“, verweist sie zugleich darauf, dass diese ,soziale Gleichheit“ eben ein (womöglich unrealisierbar bleibendes) Ideal sei. Somit gehen die politischen Gegenmaßnahmen, die von den Interviewpartnern im Kontext der Unvermeidbarkeitsdeutung gefordert werden, selten über die Gewährleistung eines Mindestlebensstandards für die Benachteiligten hinaus. Weiterreichende Forderungen nach einer Umgestaltung (oder gar Aufhebung) gesellschaftlicher Ungleichheitsverhältnisse werden aus dieser Sicht nicht formuliert. Nicht untypisch in diesem Kontext ist die Ansicht dieses Befragten:

„Gegen Ungleichheit - die ist da, da kann man nicht viel unternehmen. Die war immer da, die wird immer da sein. Was man versuchen kann, was man versuchen sollte, ist, den Leuten, die im sozialen Kontext ganz unten stehen, dass man versucht, diese Basis höher zu heben. Dass man versucht, nicht alle Leute in der Mitte zusammenzuquetschen, sondern dass man eher versucht, diesen Leuten von unten her einen höheren Lebensstandard zu gewährleisten.“ (Interview B-1)

Diese Konsequenz hat bereits Moore eindrücklich beschrieben: „Menschen neigen offenbar dazu“, so Moore (1982, S. 604 f.), „dem, was unvermeidlich scheint oder ist, Legitimität zuzusprechen [...]. Die Überwindung dieses Gefühls der Unvermeidlichkeit ist wesentlich für die Entwicklung einer politisch wirksamen moralischen Empörung. Soll dergleichen geschehen, müssen die Menschen ihre Lage als Folge menschlicher Ungerechtigkeit wahrnehmen und definieren: Eine Situation, die sie nicht ertragen müssen, können und wollen“. Ein solches Deutungsmuster, das den Befragten die gesellschaftliche „Konstruiertheit“" - und damit auch die Veränderbarkeit - sozialer Strukturen bewusst macht, wird im folgenden Abschnitt näher charakterisiert. Zudem wird gefragt, inwiefern in seinem Rahmen von den Befragten grundlegendere Forderungen nach Veränderung der Ungleichheitsstruktur artikuliert werden. 


\section{2 „Ich denke, es wird einem ein bisschen mit in die Wiege gelegt ...“-}

Deutungsmuster der Herkunftsbedingtheit sozialer Ungleichheit

Obwohl das Deutungsmuster der Unvermeidbarkeit ein dominantes Thema in den Interviews ist, nehmen die Interviewpartner zugleich eine breite Ungleichverteilung von Lebensbedingungen und -chancen nach der sozialen Herkunft wahr (vgl. auch Tab. 2 im Anhang). Darin äußert sich ein Bewusstsein für die soziale Konstruiertheit gesellschaftlicher Ungleichheitsstrukturen. Die entsprechenden Interpretationen lassen sich zu einem (konkurrierenden) Deutungsmuster der Herkunftsbedingtheit sozialer Ungleichheit verdichten, in dessen Zentrum die Betonung der Rolle der sozialen Herkunft für die Position steht, die man im Ungleichheitsgefüge einnimmt. Die von den Befragten wahrgenommenen Einflusswege der sozialen Herkunft können dabei in 1) Einflüsse der materiellen Lage der Herkunftsfamilie sowie 2) soziokulturelle Einflussfaktoren unterteilt werden (vgl. Tab. 1).

1) Der offensichtlichste Einfluss der sozialen Herkunft ist in den Augen der Interviewpartner über die materielle Lage der Herkunftsfamilie vermittelt. Die unmittelbarste Form einer solchen Beeinflussung besteht in der Vererbung materiellen Besitzes (etwa Vermögen oder Immobilien). Besonders Befragte aus unteren sozialen Klassen führen dies als einen Grund für materielle Privilegierung an. So äußert etwa eine Postzustellerin, die zugleich ergänzende Sozialleistungen bezieht, die Auffassung, eine privilegierte materielle Lage sei praktisch nur durch Erbschaften zu erreichen: „In der heutigen Zeit kann man ja nur Millionär werden, sag' ich mal, wenn man was übernommen hat, der Vater hatte vielleicht auch schon 'ne große Firma, nich'. Und jetzt der Sohn oder die Tochter, dann bleibt's in der Familie. Oder man hat vielleicht geerbt, steckt das auch noch mit in die Firma rein." (Interview C-2) Sozioökonomische Privilegierung scheint dieser Interviewpartnerin nur durch die direkte Weitergabe materieller Güter in der Familie erreichbar, kaum jedoch durch eigene Leistung: „so von Hand, ob da jetzt einer 'nen Malereibetrieb oder Kfz-Werkstatt, und dass man dann Millionär wird, kaum zu schaffen heutzutage“ (Interview C-2). Über Personen in einer privilegierten Lage denkt sie, „die werden schon Geld mitgebracht haben“. Die Bedeutung intergenerationaler Transfers von Vermögen und materiellem Besitz für die sozioökonomische Lage des Einzelnen wird auch von anderen Befragten betont. Dabei können sich in den Augen der Interviewpartner auch benachteiligte finanzielle Lagen unmittelbar aus der materiellen Situation der Herkunftsfamilie ergeben, z. B. über die Vererbung von Schulden.

Daneben verweisen die Befragten auch auf indirekte Einflüsse der materiellen Lage der Herkunftsfamilie, über die etwa bestimmte Bildungsmöglichkeiten erleichtert oder erschwert werden, was sich wiederum auf die späteren Erwerbschancen der betreffenden Personen auswirke. Kindern aus benachteiligten sozialen Lagen werden dabei geringere Chancen auf weiterführende Bildung unterstellt. ${ }^{12}$ Wiederum spielt der materielle Hintergrund in den Augen der Interviewpartner eine wichtige Rolle: So wird beispielsweise auf die Bedeutung des finanziellen Hintergrunds der Herkunftsfamilie für den Besuch qualitativ hochwertiger Kindergärten und (Privat-)Schulen oder die Möglichkeit der Finanzie-

12 Damit folgen die Wahrnehmungen der Befragten der soziologischen Ungleichheits- und Mobilitätsforschung (Kristen 2002; Müller und Pollak 2004). 
rung von Nachhilfeunterricht und Studium verwiesen. Eine ungelernte Angestellte etwa sagt:

„Wenn [...] Du jetzt 'n Kind kriegst und wirst arbeitslos, das wird das Kind ja auch merken, würde ja auch ganz anders aufwachsen, als wenn Du jetzt wirklich als Doktor arbeiten würdest in der Arztpraxis und hättst 'n bisschen mehr Geld in der Tasche. [...] Da würde ich vielleicht dem Kind noch mal Nachhilfe bezahlen, aber wenn man arbeitslos ist, nicht. [...] Das kann man als Arbeitsloser den Kindern ja nicht finanzieren." (Interview C-6)

2) Wenngleich sich solche Wahrnehmungen auch bei den Angehörigen höherer sozialer Klassen finden, ist in deren Augen die finanzielle Lage der Herkunftsfamilie nur teilweise ausschlaggebend. Wichtig(er) erscheinen ihnen mithin soziokulturelle Einflussfaktoren, über die der Zusammenhang zwischen sozialer Herkunft und Bildungschancen vermittelt werde. Besonders betont werden dabei den Eltern zugeschriebene Verhaltensweisen und Bewertungsmuster, so etwa fehlende Hilfestellung, ein Mangel an Vertrautheit mit höheren Bildungsinstitutionen oder niedrigere Bildungsambitionen. Ein Ingenieur aus der oberen Dienstklasse äußert dies folgendermaßen:

„Also, ich sag mal, vieles kann man da sicherlich [...] auch auf die Erziehung beziehungsweise auf die Eltern zurückführen, weil es ja sicherlich viele Familien gibt, die nicht so viel Wert auf Bildung legen beziehungsweise das Bildungsbewusstsein nicht so haben wie andere Eltern und da auch nicht entsprechend ihre Kinder fördern, wie's eigentlich gut wäre für sie, und da kommt dann halt eins zum andern." (Interview B-2)

Daneben verweisen andere Interviewpartner aus den höheren Klassen auch auf die mittelbaren Einflüsse bestimmter Herkunftsmilieus auf die Aspirationen der Kinder. Die im vorherigen Abschnitt zitierte selbstständige Grafikerin etwa findet, dass ,wenn man gewisse Lebensumstände nicht kennt, [...] hat man dieses Wissen nicht und kommt man da unter Umständen auch nie hin. [...] Und wenn ich Dinge nicht kenne und von denen nichts weiß, dann zieht's mich da auch nicht hin“ (Interview D-4). Somit sind Bildungschancen in der deutschen Gesellschaft in den Augen der Interviewpartner zwar ungleich verteilt, doch sind für Befragte aus höheren sozialen Klassen hierfür nicht allein materielle Umstände, sondern auch die Denkmuster und Verhaltensweisen der Eltern verantwortlich. Damit wird die Handlungsmacht des Elternhauses akzentuiert und die Verantwortungszuschreibung für benachteiligte soziale Lagen verschoben: Ungleichheit erscheint zwar immer noch als herkunftsbedingt (weil durch die Familie beeinflusst), doch die materielle Lage des Elternhauses ist kein hinreichender Grund für die Abstinenz der Kinder von höherer Bildung, da diese auch von den Ambitionen der Eltern abhänge.

Stellt man dem gegenüber, wie sich in den Augen der Befragten eine ,privilegierte“ soziale Herkunft auswirke, so fällt auf, dass diese Einflusswege viel eher den Charakter mehr oder minder bewusster „Weichenstellungen“ annehmen. Dies wird sowohl in den biografischen Schilderungen von Interviewpartnern aus oberen Klassen wie auch in ihren Ausführungen zur Förderung der eigenen Kinder deutlich. Dabei sind sich die Eltern (und insbesondere die befragten Mütter) aus den höheren Klassen ihres eigenen Einflusses und ihrer Handlungsmacht durchaus bewusst. Entscheidungen beispielsweise zur Bildungs- 
laufbahn ihrer Kinder gewinnen so einen „strategischen“ Charakter. ${ }^{13}$ Dies impliziert auch ein größeres Bewusstsein für die gesellschaftlichen Ursachen von Ungleichheitsstrukturen in dieser Gruppe. Sehr klar kommt dies in den Ausführungen einer selbstständigen Therapeutin zum Ausdruck. Sowohl beim Vergleich der Bildungschancen von Kindern aus ,schwächer gestellten“ Verhältnissen mit denen ihres eigenen Sohnes als auch bei der Reflexion ihrer eigenen „sozialen Laufbahn“ (Bourdieu) tritt dies zutage:

„Das heißt, wenn ich da hingucke, das baut sich ja auf, nich’, diese Kinder kommen anders zur Schule. Die gehen auch in 'ne andere Grundschule als mein Sohn. Weil ich auch da 'ne Wahl treffe und sage, ich geh' nicht in jede Grundschule [...].“ (Interview D-3)

„Und dann, ich seh’ das so, dass ich da bin, wo ich bin, das verdanke ich meiner Leistung, aber das verdanke ich auch ganz vielen anderen Faktoren. Also beispielsweise meiner Herkunftsfamilie, da wo ich herkomme, die Art und Weise, wie ich aufwachsen konnte in meiner Familie, wie meine Eltern uns gefördert haben. [...] [D]adurch war es mir viel einfacher, durch die Schule zu gehen, als anderen Kindern, die nicht so aufgewachsen sind. [...] Aber es ist doch, also eher als Teil von etwas bin ich in 'ne günstige Situation gekommen.“ (Interview D-3)

Hier wird zum einen deutlich, wie sehr sich diese Befragte ihres eigenen Einflusses auf die Entwicklung ihres Sohnes bewusst ist (,weil ich auch da 'ne Wahl treffe“), und dass dieser andere - und auch: bessere - Bildungschancen habe als Kinder aus benachteiligten Familien. Gleichzeitig sieht sie, dass die elterliche Förderung auch ihre eigene Laufbahn geprägt hat. Dies kommt sehr deutlich zum Vorschein, als sie ihre eigene Biografie resümiert. Hier werden Impulse, sich die eigene privilegierte Lage einzig aufgrund von Leistung zuzuschreiben, deutlich abgeschwächt und die soziale Prägung der eigenen sozialen Laufbahn betont (,eher als Teil von etwas bin ich in 'ne günstige Situation gekommen").

Insgesamt impliziert das Deutungsmuster der Herkunftsbedingtheit ein größeres Bewusstsein für die sozialen Grundlagen gesellschaftlicher Ungleichheitsstrukturen sowie die eigene Position darin, wenngleich mit unterschiedlichen schichtspezifischen Akzentuierungen: Während die Interviewpartner aus den unteren Klassen eher auf die materiellen Umstände der Herkunftsfamilie abstellen, ist für Befragte aus den höheren Klassen eher das soziokulturelle Umfeld entscheidend. In beiden Fällen scheint jedoch eine Voraussetzung für ungleichheitskritische(re) Sichtweisen und ein größeres Potenzial für De-Legitimierungen gegeben als bei der Unvermeidbarkeitsdeutung.

Dieses artikuliert sich insbesondere in einer normativen Orientierung am Ideal der Chancengleichheit, die bei den Angehörigen höherer Klassen jedoch stärker ausgeprägt ist als bei den Angehörigen niedriger Klassen. Letztere konstatieren zwar ebenso wie Interviewpartner aus den höheren Klassen einen faktischen Mangel an Chancengleichheit, doch stellt diese für sie kaum einen normativen Bezugspunkt dar (vgl. näher Sach-

13 Vgl. die Befunde bei Lareau (2002), denen zufolge Eltern aus oberen Klassen einen Erziehungsstil der „konzertierten Kultivierung“ verfolgen und die Laufbahnen ihrer Kinder gezielt fördern und beeinflussen. Eltern aus unteren Klassen dagegen folgen einem Ansatz des ,natürlichen Wachstums“, bei dem weniger Wert auf die gezielte Förderung spezifischer Talente gelegt wird. 
weh 2010, S. 251 ff.). Für die Befragten aus den oberen Klassen bildet Chancengleichheit dagegen ein normatives Ideal, auf das sie sich bei der Kritik von Ungleichheitsverhältnissen berufen und dessen Beförderung sie als politische Aufgabe ansehen. So äußert eine selbstständige Grafikdesignerin etwa die Ansicht, „,dass eben nicht jeder die gleichen Möglichkeiten hat, das heißt gewisse Dinge bleiben einem verwehrt. Und das ist immer 'n Problem, finde ich“ (Interview D-4). Entsprechend bedeutet „Gerechtigkeit“ für sie, „dass jeder auch die gleichen Chancen haben sollte“ (Interview D-4). Somit scheint das Deutungsmuster der Herkunftsbedingtheit im Einklang mit dem eingangs skizzierten Erwartungen des aufklärerischen Common Sense an die Wahrnehmung sozialer Ungleichheit zu stehen.

\subsection{Konkurrierende oder koexistierende Deutungsmuster sozialer Ungleichheit?}

An dieser Stelle lassen sich die empirischen Befunde vorläufig zusammenfassen: Es wurden zwei verschiedene alltagsweltliche Erklärungsmuster sozialer Ungleichheit rekonstruiert, die sich darin unterscheiden, inwieweit sie Ungleichheit als ein von Menschen gemachtes, sozial verursachtes Konstrukt (Herkunftsbedingtheit sozialer Ungleichheit) oder einen notwendigen Ausdruck gesellschaftlicher Ordnung (Unvermeidbarkeit sozialer Ungleichheit) betrachten. Beide Deutungsmuster legen unterschiedliche normative Orientierungen aufseiten der Befragten nahe und haben somit tendenziell gegensätzliche Implikationen für die Legitimation von Ungleichheit. Während die Unvermeidbarkeitsdeutung mit einer eher legitimierenden Haltung einhergeht, legt die Herkunftsdeutung eher kritische Bewertungen und eine Orientierung am Grundsatz der Chancengleichheit nahe. Zudem wurde deutlich, dass beide Deutungsmuster sowohl von Befragten aus oberen als auch aus unteren Klassenlagen zur alltagsweltlichen Erklärung von Ungleichheit herangezogen werden - obgleich beim Deutungsmuster der Herkunftsbedingtheit eine recht deutliche klassenspezifische Modulation dieses Grundmusters besteht (vgl. hierzu noch Abschn. 6). Es stellt sich daher die Frage, inwiefern diese auf einer logischen Ebene widersprüchlichen Deutungsmuster auch in der Praxis, d. h. bei der alltagsweltlichen Erklärung sozialer Ungleichheit, miteinander konkurrierende und sich wechselseitig ausschließende Deutungsmuster darstellen.

Die Tatsache, dass beide Deutungsmuster klassenübergreifend und von ein- und denselben Befragten angewandt werden, verweist darauf, dass es sich hier nur bedingt um miteinander konkurrierende Deutungsmuster zu handeln scheint. Vielmehr ist von einer Koexistenz der beiden Deutungsmuster auszugehen, die sich in bestimmten Hinsichten ergänzen und - trotz ihrer latenten Widersprüchlichkeit - von den Befragten in ihren alltagsweltlichen Erklärungen auch kombiniert und miteinander in Einklang gebracht werden. Besonders deutlich wird dies in den Bewertungen der Möglichkeit sozialer Aufwärtsmobilität (inter- oder intragenerational) aus benachteiligten Verhältnissen durch die Interviewpartner. Nicht nur die Wahrnehmung der Unvermeidbarkeit von Ungleichheit, sondern auch einer herkunftsbedingten Benachteiligung von Kindern aus unteren Klassen legen die Vermutung nahe, dass die Befragten die Möglichkeit von Aufwärtsmobilität aus benachteiligten Lagen eher pessimistisch einschätzen sollten. Überraschend ist daher, dass dies keineswegs durchgehend so gesehen wird. Stattdessen betonen die Interviewpartner die Möglichkeit, sich „,von unten hochzuarbeiten“. Ein sozialer Aufstieg sei dabei 
vor allem von bestimmten Persönlichkeitseigenschaften - etwa Hartnäckigkeit, Zielstrebigkeit, Disziplin und Durchsetzungsvermögen - abhängig. Eine Personalberaterin, selbst Aufsteigerin, erläutert dies wie folgt:

„Man muss wahnsinnig diszipliniert sein, einen ganz starken Willen haben, und, ja, ich würde sagen Durchsetzungsvermögen. Und vielleicht auch den Glauben an sich. Der ganz starke Glaube an sich, man schafft das, ,Du schaffst das' . [...] Das ist aber wiederum was, das hängt glaube ich zu fünfzig Prozent von der Persönlichkeit ab und zu fünfzig Prozent vom Elternhaus. Und das ist unabhängig davon, ob die Eltern Akademiker sind oder wohlhabend sind.“ (Interview D-2)

Die Persönlichkeitsmerkmale, die als Voraussetzung für den sozialen Aufstieg angesehen werden, erscheinen zwar durch die Familie geprägt, nicht aber durch deren soziale Lage - und werden somit als universell unterstellt. ${ }^{14}$ Dadurch kann die Überzeugung gestützt werden, dass trotz niedriger sozialer Herkunft ein individueller Aufstieg möglich sei, wenngleich er auch mit mehr Anstrengung, Disziplin und Verzichtsbereitschaft verbunden ist als für Kinder aus oberen sozialen Klassen. Damit wird einerseits eine zentrale Variante der Unvermeidbarkeitsdeutung aufgegriffen (Ungleichheit als Folge individueller Eigenschaften und Anlagen), andererseits aber der Einfluss der sozialen Herkunft auf Ungleichheitsstrukturen nicht geleugnet. So ist es möglich, dass ein- und dieselben Personen auf beide Deutungsmuster zur Erklärung von Ungleichheit in der deutschen Gesellschaft zurückgreifen können. ${ }^{15}$ Das ungleichheitskritische Potenzial der Herkunftsdeutung, das im vorherigen Abschnitt illustriert wurde, wird so unterlaufen.

\section{Diskussion}

Der Ausgangspunkt dieses Artikels war die Frage, inwieweit die im eingangs skizzierten aufklärerischen Common Sense der Ungleichheitsforschung implizierten Annahmen - in der Moderne habe sich eine Sichtweise herausgebildet, die Ungleichheit als gesellschaftlich verursacht und damit als legitimationsbedürftig betrachte - mit Blick auf die alltagsweltlichen Ungleichheitsdeutungen der Gesellschaftsmitglieder realistisch sind. Die empirischen Befunde legen den Schluss nahe, dass Ungleichheitsstrukturen nicht in dem Maße als gesellschaftlich verursacht angesehen werden, wie vom aufklärerischen Common Sense erwartet. Zwar rückt das Deutungsmuster der Herkunftsbedingtheit sozialer Ungleichheit die gesellschaftlichen Konstruktionsprozesse von Ungleichheit in den Vordergrund und legt damit teils auch ungleichheitskritische normative Orientierungen bei den Interviewpartnern nahe. Parallel dazu existiert jedoch ein Deutungsmuster der

14 Diese Deutung findet sich nicht nur bei aufwärtsmobilen Befragten, sondern stellt eine geteilte Auffassung unter den Interviewpartnern dar (vgl. Abschn. 5.1).

15 Vgl. auch die Ergebnisse bei Kluegel und Smith (1986), denen zufolge ein Teil der von ihnen befragten US-Amerikaner sowohl individualistische als auch strukturalistische Ungleichheitsgründe anführen. Die Autoren interpretieren diesen Befund ebenfalls so, dass eine solche Kompromissposition die Wirksamkeit struktureller Barrieren anerkenne, gleichzeitig aber darauf hingewiesen werde, dass diese strukturellen Grenzen durch individuelle Anstrengung überwunden werden könnten (ebd., S. 87 f.). 
Unvermeidbarkeit sozialer Ungleichheit, das Ungleichheit gerade nicht als gesellschaftliches Konstrukt, sondern als Folge der menschlichen Natur oder einer unausweichlichen Begleiterscheinung gesellschaftlicher Ordnung betrachtet. Somit dauern nach wie vor ,voraufklärerische“ Sichtweisen fort, die sich auf naturalistische oder essenzialistische Erklärungsmuster sozialer Ungleichheit stützen. Diese entlasten die Befragten von einem Rechtfertigungsdruck, der virulenter wäre, wenn Ungleichheit als sozial verursacht angesehen würde. Indem die Unvermeidbarkeitsdeutung die sozialen Ursachen von Ungleichheit ausblendet, trägt sie zur Legitimation von Ungleichheit bei. Dies wird in den entsprechenden normativen Orientierungen der Befragten deutlich, die sich im Rahmen der Unvermeidbarkeitsdeutung eine egalitärere Gesellschaft nur schwer vorstellen können. Für die Ungleichheitstheorie bedeutet dies, dass sich die im aufklärerischen Hintergrundkonsens implizierten Annahmen über den Wandel von Ungleichheitsverständnissen als eine sozialwissenschaftliche Projektion in die Gesellschaft hinein erweisen, an der die hier dargestellten Interviewdaten Zweifel wecken.

Weiterhin zeigen die empirischen Befunde, dass beide Deutungsmuster trotz ihrer Widersprüchlichkeit auf einer logischen Ebene - Ungleichheit als unvermeidbares, notwendiges Faktum vs. Ungleichheit als gesellschaftliches, von Menschen geschaffenes Konstrukt - miteinander vereinbar zu sein scheinen und von ein- und denselben Befragten gleichzeitig angewandt werden. Es handelt sich somit nicht um im strengen Sinne konkurrierende, sondern vielmehr um miteinander koexistierende Deutungsmuster. Die argumentative Verbindung, mittels derer die Befragten beide Deutungen miteinander verknüpfen, bildet der Verweis auf die Möglichkeit eines sozialen Aufstiegs aus benachteiligten Verhältnissen beim Besitz der ,richtigen“ Persönlichkeitseigenschaften. Dieser greift eine zentrale Variante der Unvermeidbarkeitsdeutung auf (Ungleichheit als Folge individueller Persönlichkeitseigenschaften), ohne den Einfluss der sozialen Herkunft zu leugnen. Die ungleichheitskritischen Impulse der Herkunftsdeutung werden so konterkariert.

Keines der beiden Erklärungsmuster wird zudem ausschließlich von Befragten einer bestimmten sozialstrukturellen Lage vertreten. Anstelle klassenspezifischer Deutungsmuster finden sich vielmehr klassenspezifische Variationen bzw. Modulationen ein- und desselben Deutungsmusters. Der übergeordnete Tenor eines Deutungsmusters wird dabei von Befragten aus verschiedenen Klassen geteilt, die konkrete inhaltliche Gestalt der Interpretationen unterscheidet sich jedoch klassenspezifisch. Dies zeigt sich beim Deutungsmuster der Herkunftsbedingtheit: Sowohl Befragte aus privilegierten als auch aus benachteiligten Klassen sehen in der sozialen Herkunft eine wichtige Ungleichheitsursache. Ihre Wahrnehmungen über die konkrete Wirkung der sozialen Herkunft unterscheiden sich jedoch. Während Befragte aus benachteiligten Lagen hier stärker auf die materielle Lage der Herkunftsfamilie abstellen, betonen die Interviewpartner aus privilegierten Lagen die mit dem familiären Umfeld verbundenen soziokulturellen Einflüsse. Die Bezugnahme auf fehlende finanzielle Mittel der Herkunftsfamilie seitens der Befragten aus den unteren Klassen ist angesichts ihrer materiellen Situation nicht erstaunlich. Den Hintergrund für die stärkere Akzentuierung der soziokulturellen Einflüsse durch die Befragten aus den oberen Klassen bildet dagegen deren bewusste Intervention in die Laufbahnen ihrer Kinder, die ihnen die Handlungsmacht des Elternhauses und die Gestaltbarkeit individueller Lebenschancen vor Augen führt. Die klassenspezifischen Variationen der Herkunftsdeutung scheinen somit in der differenziellen Erfahrungs- 
gebundenheit einzelner Wahrnehmungen begründet zu sein. Je nachdem, ob und wie sehr bestimmte Erfahrungen in der jeweiligen Lebenswelt einer Gruppe verankert sind, beeinflusst dies auch ihre ungleichheitsbezogenen Deutungen. ${ }^{16}$ Damit bilden die in diesem Artikel rekonstruierten Deutungsmuster der Unvermeidbarkeit und der Herkunftsbedingtheit sozialer Ungleichheit interpretative Repertoires von klassenübergreifender Reichweite, die in ihrer konkreten inhaltlichen Ausformung an die konkreten Umstände der Individuen angepasst werden.

Angesichts der Datengrundlage dieser Studie können keine Rückschlüsse auf die gesamtgesellschaftliche Reichweite und Verbreitung der rekonstruierten Deutungsmuster gezogen werden. Allerdings geben die Ergebnisse einer früheren Umfrage von Kluegel und Smith (1986, S. 104 ff.) in den USA Hinweise auf ein Einstellungsmuster, das dem hier rekonstruierten Deutungsmuster der Unvermeidbarkeit nahekommt. So vertritt etwa eine Mehrheit der von Kluegel und Smith befragten US-Amerikaner die Auffassung, Einkommensunterschiede könnten nicht verringert werden, weil es immer Unterschiede in den Fähigkeiten und Begabungen der Menschen gebe (85\%) und es in der menschlichen Natur liege, mehr als andere haben zu wollen (81\%). Wenngleich diese Befunde aufgrund historischer und soziokultureller Unterschiede nicht umstandslos auf Deutschland übertragen werden können, so verweisen sie dennoch darauf, dass es lohnend wäre, wenn sich künftige Forschungsanstrengungen - gerade auch solche quantitativ-vergleichender Art - der genaueren Bestimmung der Verbreitung der Unvermeidbarkeitsdeutung (und ihrer legitimatorischen Konsequenzen) annähmen. Mit Blick auf das methodische Vorgehen der Umfrageforschung scheint es angesichts der diskutierten Befunde geboten, Items in stärkerem Maße aus den Alltagswissensbeständen der Gesellschaftsmitglieder (und nicht unter Bezugnahme auf soziologische Theorien oder Ad-hoc-Überlegungen) zu formulieren. Dieses Verfahren könnte das empirische Wissen über das inhaltliche Spektrum von Ungleichheitsdeutungen erheblich erweitern und damit auch zur Selbstvergewisserung der Ungleichheitssoziologie über ihre eigenen theoretischen Vorannahmen beitragen.

16 Auch die fehlenden Geschlechterunterschiede in den Deutungen sprechen dafür, dass unterschiedliche Ungleichheitsdeutungen eher mit (sozialstrukturell geprägten) lebensweltlich-biografischen Erfahrungen zusammenhängen als mit der bloßen Gruppenzugehörigkeit. 


\section{Anhang}

Tab. 2: Wahrnehmung von Ungleichheitsgründen in Deutschland

1. Schichtunterschiede: „In Deutschland gibt es noch große Unterschiede zwischen den sozialen Schichten, und was man im Leben erreichen kann, hängt im Wesentlichen davon ab, aus welchem Elternhaus man kommt"

Westdeutschland $\%$,stimme voll zu“ und „stimme eher zu“

Ostdeutschland 55

Gesamtdeutschland 68,8 57,5

2. Offene Gesellschaft: „Deutschland ist eine offene Gesellschaft. Was man im Leben erreicht, hängt nicht mehr vom Elternhaus ab, aus dem man kommt, sondern von den Fähigkeiten, die man hat, und der Bildung, die man erwirbt" $\%$,stimme voll zu“ und „stimme eher zu“"

Westdeutschland 81,7

Ostdeutschland 65,6

Gesamtdeutschland 78,7

3. Ungleichheit nutzt den Reichen: „Die sozialen Unterschiede bleiben bestehen, weil sie den Reichen und Mächtigen nützen“"

$$
\% \text {,stimme stark zu“ und ,stimme zu““ }
$$

Westdeutschland 73,6

Ostdeutschland 83,1

Gesamtdeutschland 75,5

Items 1 und 2 ALLBUS 2004 (ZA-Nr. 3762), Item 3 ALLBUS 2000 (ZA-Nr. 3450), eigene Berechnungen. Antwortkategorien der Items 1 und 2: „stimme voll zu“, „stimme eher zu“, „stimme eher nicht zu“, „stimme überhaupt nicht zu“, Antwortkategorien Item 3: „stimme stark zu“, „stimme zu“, ,,weder noch“, ,stimme nicht zu“, ,stimme überhaupt nicht zu“

\section{Literatur}

Barlösius, E. (2005). Die Macht der Repräsentation. Common Sense über soziale Ungleichheiten. Wiesbaden: VS Verlag für Sozialwissenschaften.

Berger, J. (2004). Über den Ursprung der Ungleichheit unter den Menschen. Vergangenheit und Gegenwart einer soziologischen Schlüsselfrage. Zeitschrift für Soziologie, 33, 354-374.

Berger, P. A. (1988). Die Herstellung sozialer Klassifikationen. Methodische Probleme der Ungleichheitsforschung. Leviathan, 16, 501-520.

Boltanski, L., \& Thévenot, L. (1999). The sociology of critical capacity. European Journal of Social Theory, 2, 359-377.

Bottero, W. (2005). Stratification. Social division and inequality. London: Routledge.

Burzan, N. (2004). Soziale Ungleichheit. Eine Einführung in die zentralen Theorien. Wiesbaden: VS Verlag für Sozialwissenschaften. 
Dahrendorf, R. (1974). Über den Ursprung der Ungleichheit unter den Menschen. In R. Dahrendorf, Pfade aus Utopia. Zur Theorie und Methode der Soziologie (S. 352-379). München: Piper.

Dux, G. (2008). Warum denn Gerechtigkeit? Die Logik des Kapitals. Die Politik im Widerstreit mit der Ökonomie. Weilerswist: Velbrück Wissenschaft.

Eder, K. (1990). Gleichheitsdiskurs und soziale Ungleichheit. In H. Haferkamp (Hrsg.), Sozialstruktur und Kultur (S. 177-208). Frankfurt a. M.: Suhrkamp.

Erikson, R., \& Goldthorpe, J. H. (1992). The constant flux. A study of class mobility in industrial societies. Oxford: Clarendon Press.

Feagin, J. R. (1975). Subordinating the poor. Welfare and American beliefs. Englewood Cliffs: Prentice Hall.

Giesen, B. (1987). Natürliche Ungleichheit, Soziale Ungleichheit, Ideale Gleichheit: Zur Evolution von Deutungsmustern sozialer Ungleichheit. In B. Giesen \& H. Haferkamp (Hrsg.), Soziologie der sozialen Ungleichheit (S. 314-345). Opladen: Westdeutscher Verlag.

Gottschall, K. (2000). Soziale Ungleichheit und Geschlecht. Kontinuitäten und Brüche, Sackgassen und Erkenntnispotentiale im deutschen soziologischen Diskurs. Opladen: Leske+Budrich.

Hochschild, J. L. (1981). What's fair? American beliefs about distributive justice. Cambridge: Harvard University Press.

Hopf, C. (1978). Die Pseudo-Exploration. Überlegungen zur Technik qualitativer Interviews in der Sozialforschung. Zeitschrift für Soziologie, 7, 97-115.

Hyman, H. H. (1966). The value system of different classes. A social psychological contribution to the analysis of stratification. In R. Bendix \& S. M. Lipset (Hrsg.), Class, status, and power. Social stratification in comparative perspective (S. 488-499). New York: The Free Press.

Kelle, U., \& Kluge, S. (1999). Vom Einzelfall zum Typus. Fallvergleich und Fallkontrastierung in der qualitativen Sozialforschung. Opladen: Leske+Budrich.

Kluegel, J. R., \& Smith, E. (1986). Beliefs about inequality. American's view of what is and what ought to be. New York: Aldine de Gruyter.

Kohn, M. (1977). Class and conformity. A study in values. Chicago: University of Chicago Press.

Kohn, M. (1980). Job complexity and adult personality. In N. J. Smelser \& E. H. Erikson (Hrsg.), Themes of work and love in adulthood (S. 193-210). Cambridge: Harvard University Press.

Koller, P. (1987). Die Rechtfertigung und Kritik sozialer Ungleichheit. Österreichische Zeitschrift für Soziologie, 12, 4-26.

Kreckel, R. (2004). Politische Soziologie der sozialen Ungleichheit. Frankfurt a. M.: Campus.

Kristen, C. (2002). Hauptschule, Realschule oder Gymnasium? Ethnische Unterschiede am ersten Bildungsübergang. Kölner Zeitschrift für Soziologie und Sozialpsychologie, 54, 534-552.

Kuckartz, U. (2006). Computerunterstütze Analyse qualitativer Daten. In A. Diekmann (Hrsg.), Methoden der Sozialforschung. Sonderheft 44 der Kölner Zeitschrift für Soziologie und Sozialpsychologie (S. 453-478). Wiesbaden: VS Verlag für Sozialwissenschaften.

Lamont, M. (2000). The dignity of working men. Morality and the boundaries of race, class, and immigration. Cambridge: Harvard University Press.

Lareau, A. (2002). Invisible inequality. Social class and childrearing in black and white families. American Sociological Review, 67, 747-776.

Mau, S. (2004). Moralökonomie. Eine konzeptionelle Bestimmung aus ungleichheitssoziologischer Sicht. In P. A. Berger \& V. H. Schmidt (Hrsg.), Welche Gleichheit, welche Ungleichheit? Grundlagen der Ungleichheitsforschung (S. 165-190). Wiesbaden: VS Verlag für Sozialwissenschaften.

Mayer, K.-U., Kraus, V., \& Schmidt, P. (1992). Opportunity and inequality: Exploratory analyses of the structure of attitudes toward stratification in West Germany. In F. C. Turner (Hrsg.), Social mobility and political attitudes (S. 51-78). New Brunswick: Transaction Publishers.

Meuser, M., \& Sackmann, R. (1992). Zur Einführung: Deutungsmusteransatz und empirische Wissenssoziologie. In M. Meuser \& R. Sackmann (Hrsg.), Analyse sozialer Deutungsmuster. Beiträge zur empirischen Wissenssoziologie (S. 9-37). Pfaffenweiler: Centaurus. 
Miller, D. (1999). Principles of social justice. Cambridge: Harvard University Press.

Müller, W., \& Pollak, R. (2004). Warum gibt es so wenige Arbeiterkinder in Deutschlands Universitäten? In R. Becker \& W. Lauterbach (Hrsg.), Bildung als Privileg? Erklärungen und Befunde zu den Ursachen der Bildungsungleichheit (S. 311-352). Wiesbaden: VS Verlag für Sozialwissenschaften.

Moore, B. (1982). Ungerechtigkeit. Die sozialen Ursachen von Unterordnung und Widerstand. Frankfurt a. M.: Suhrkamp.

Newman, K. S. (1999). Falling from grace. Downward mobility in an age of affluence. Berkeley: University of California Press.

Nollmann, G. (2003). Die stille Umverteilung. Budgetierung als Transmissionsriemen für die Verschärfung von Einkommensungleichheit. Kölner Zeitschrift für Soziologie und Sozialpsychologie, 55, 500-520.

Oevermann, U. (2001a). Die Struktur sozialer Deutungsmuster - Versuch einer Aktualisierung. Sozialer Sinn, 2, 35-81.

Oevermann, U. (2001b). Kommentar zu Christine Plaß und Michael Schetsche: „Grundzüge einer wissenssoziologischen Theorie sozialer Deutungsmuster“. Sozialer Sinn, 2, 537-546.

Oevermann, U. (2001c). Zur Analyse der Struktur von sozialen Deutungsmustern. Sozialer Sinn, $2,3-33$.

Orton, M., \& Rowlingson, K. (2007). Public attitudes to economic inequality. York: Joseph Rowntree Foundation.

Parsons, T. (1970). Equality and inequality in modern society, or social stratification revisited. In E. O. Laumann (Hrsg.), Social stratification: Theory and research for the 1970s (S. 13-72). Indianapolis: Bobbs-Merrill Co.

Rodman, H. (1963). The lower class value stretch. Social Forces, 42, 205-215.

Rousseau, J.-J. (1984). Diskurs über die Ungleichheit unter den Menschen. Paderborn: Ferdinand Schöningh.

Sachweh, P. (2010). Deutungsmuster sozialer Ungleichheit. Wahrnehmung und Legitimation gesellschaftlicher Privilegierung und Benachteiligung. Frankfurt a. M.: Campus.

Schmidt, V. H. (2000). Bedingte Gerechtigkeit. Soziologische Analysen und philosophische Theorien. Frankfurt a. M.: Campus.

Schmidt, V. H. (2004). Ungleichgewichtige Ungleichheiten. In P. A. Berger \& V. H. Schmidt (Hrsg.), Welche Gleichheit, welche Ungleichheit? Grundlagen der Ungleichheitsforschung (S. 73-92). Wiesbaden: VS Verlag für Sozialwissenschaften.

Schütz, A. (1964). Equality and the meaning structure of the social world. In A. Schütz, Collected papers. Studies in social theory. Volume II (S. 226-273). The Hague: Martinus Nijhoff.

Schütz, A. (1974). Der sinnhafte Aufbau der sozialen Welt. Eine Einleitung in die verstehende Soziologie. Frankfurt a. M.: Suhrkamp.

Schwinn, T. (2007). Soziale Ungleichheit. Bielefeld: transcript.

Solga, H., Berger, P. A., \& Powell, J. (2009). Soziale Ungleichheit - Kein Schnee von gestern! Eine Einführung. In H. Solga, J. Powell, \& P. A. Berger (Hrsg.), Soziale Ungleichheit. Klassische Texte zur Sozialstrukturanalyse (S. 11-45). Frankfurt a. M.: Campus.

Svallfors, S. (2006). The moral economy of class. Class and attitudes in comparative perspective. Stanford: Stanford University Press.

Terpe, S. (2009). Ungerechtigkeit und Duldung. Die Deutung sozialer Ungleichheit und das Ausbleiben von Protest. Konstanz: UVK.

Ullrich, C. G. (1999). Deutungsmusteranalyse und diskursives Interview. Zeitschrift für Soziologie, $28,429-447$.

van Oorschot, W., \& Halman, L. (2000). Blame or fate, individual or social? An international comparison of popular explanations of poverty. European Societies, 2, 1-28.

Wegener, B. (1992). Gerechtigkeitsforschung und Legitimationsnormen. Zeitschrift für Soziologie, 21, 269-283. 
Wegener, B., \& Liebig, S. (1993). Eine Grid-Group-Analyse sozialer Gerechtigkeit. Die neuen und alten Bundesländer im Vergleich. Kölner Zeitschrift für Soziologie und Sozialpsychologie, 45, $668-690$.

Will, J. A. (1993). The dimensions of poverty: Public perceptions of the deserving poor. Social Science Research, 22, 312-332.

Patrick Sachweh, geb. 1979. Dr. rer. pol., Akademischer Rat a. Z. an der Goethe-Universität Frankfurt a. M. Forschungsschwerpunkte: Soziale Ungleichheit, vergleichende Wohlfahrtsstaatsforschung und Sozialpolitik, Wirtschaftssoziologie, Methoden der empirischen Sozialforschung. Ausgewählte Veröffentlichungen: Deutungsmuster sozialer Ungleichheit. Wahrnehmung und Legitimation gesellschaftlicher Privilegierung und Benachteiligung, 2010; (mit S. Olafsdottir) The welfare state and equality? Stratification realities and aspirations in three welfare regimes, in: European Sociological Review, Advance Access doi: 10.1093/esr/jcq055, 2010; (mit C. Ullrich und B. Christoph) Die gesellschaftliche Akzeptanz der Sozialhilfe. Eine Untersuchung aus moralökonomischer Perspektive, in: Kölner Zeitschrift für Soziologie und Sozialpsychologie 58, 2006. 